\title{
Sprache als immanenter Teil der Rechtsordnung
}

- Linguistische, rechtspraktische und verfassungsrechtliche Anmerkungen zur Zulassung von Englisch als Gerichtssprache an Kammern für internationale Handelssachen bei den Landgerichten -

\section{Einleitung}

In der deutschen Politik sind derzeit zwei auf den ersten Blick gegensätzliche Tendenzen zu beobachten. Während auf der einen Seite seit langem versucht wird, die deutsche Sprache als nationalen Kulturschatz gegen Fremdsprachen zu schützen, ${ }^{1}$ wird in jüngster Zeit andererseits unter den Schlagworten, globaler Wettbewerb“ und ,Standort Deutschland' der Einsatz der englischen Sprache in vor allem (juristischer) Ausbildung ${ }^{2}$ und neuerdings bei staatlichen Aufgaben gefördert. Die letztgenannte Tendenz erreicht ihren vorläufigen Höhepunkt im Entwurf des Gesetzes zur Einführung von Kammern für internationale Handelssachen (KfiHG) bei den Landgerichten, an denen Englisch optional als Gerichtssprache zugelassen werden soll. ${ }^{3}$ Zur Unterstützung und als Beleg der praktischen Durchführbarkeit startete im Oberlandesgerichtsbezirk Köln Anfang des Jahres ein von der Rechtsanwaltskammer Köln öffentlichkeitswirksam inszeniertes Modellprojekt. $^{4}$

Der von den Ländern Hamburg und Nordrhein-Westfalen in den Bundesrat eingebrachte Gesetzentwurf sieht vor, dass an den Kammern für internationale Handelssachen in Abweichung vom bisherigen $\S 184$ GVG das gesamte gerichtliche Verfahren in Englisch durchgeführt werden kann. ${ }^{5}$ Sowohl die Verhandlung kann dabei auf Englisch erfolgen. Auch Protokoll und Urteil selbst können in englischer Sprache abgefasst werden. ${ }^{6}$ Internationale Handelssachen werden als Rechtsstreitigkeiten definiert, die einen internationalen Bezug haben und in denen die Parteien übereinstimmend vereinbaren, dass die Verhandlung in englischer Sprache erfolgen soll. ${ }^{7}$

Durch die Einführung von Englisch als Gerichtssprache soll gewährleistet werden, dass der Gerichtsstandort Deutschland auch international Anerkennung erlangt. ${ }^{8} \mathrm{Ob}$ -

1 Dreesen, Staatsziel „Deutsch“. Die deutsche Sprache im Grundgesetz aus verfassungspolitischer Perspektive, Aptum Zeitschrift für Sprachkritik und Sprachkultur 2010, Heft 1, S. 37.

2 Georgen/Schulze, Fremdsprachen und Übersetzen im Rechtsstudium, in: de Groot/Schulze (Hrsg.), Recht und Übersetzen, 1999, S. 67.

3 BR Drs. 42/10 vom 7. Mai 2010 und BT Drs. 17/2163 vom 16. Juni 2010.

4 Pressemitteilung des OLG Köln vom 13.1.2010, http://www.olg-koeln.nrw.de/presse/archiv/ archiv_2010/005 01-13 einladung-pressegespr-englisch.pdf (abgerufen am 9.9.2010); FAZ Nr. 9 vom 9.1.2010; Hoppe, Englisch als Verfahrenssprache - Möglichkeiten de lega lata und de lege ferenda, Praxis des internationalen Privat- und Verfahrensrechts, 2010, S. 373.

5 BR Drs. 42/10, S. 7; zum weiteren Inhalt des Gesetzesentwurfs vgl. Müller-Piepenkötter, Englisch als Gerichtssprache, Deutsche Richterzeitung 2010, S. 2 ff.

6 BR Drs. $42 / 10$, S. 7.

7 BR Drs. $42 / 10$, S. 3.

8 BR Drs. $42 / 10$, S. 6. 
wohl das deutsche Recht hinsichtlich seiner Effizienz, seiner Leistungsfähigkeit sowie seiner niedrigen Kosten Vorzüge habe und daher auch international hohe Anerkennung genieße, werde es aufgrund der Ausschließlichkeit der Verfahrenssprache Deutsch bei der Rechtswahl durch die Parteien nicht berücksichtigt. ${ }^{9}$ Aufgrund dessen würden bedeutende wirtschaftsrechtliche Streitigkeiten entweder im Ausland oder vor Schiedsgerichten ausgetragen, was sowohl den Gerichtsstandort Deutschland als auch deutsche Unternehmen beeinträchtige. ${ }^{10}$ Die Verbreitung des deutschen Rechts soll nach der Begründung zum Gesetzentwurf begünstigen, dass das deutsche Recht international an Bedeutung gewinnt und deutsche Unternehmen eine erhöhte Rechtssicherheit im internationalen Wirtschaftsverkehr erfahren. ${ }^{11}$ Ein Sprachbruch zwischen (ganz überwiegend englischer) Vertrags- und deutscher Verfahrenssprache behindere dagegen die Klärung von Auslegungszweifeln. ${ }^{12}$

Die Debatte um den Gesetzentwurf im Plenum des Bundesrats war ausnahmslos zustimmend; ${ }^{13}$ die juristische Debatte zum Gesetzesentwurf zeigt neben vereinzelten ablehnenden Kommentaren ${ }^{14}$ entweder völlige ${ }^{15}$ oder eingeschränkte Zustimmung. ${ }^{16}$ Die folgende Auseinandersetzung mit dem Gesetzentwurf widmet sich der unzureichend behandelten linguistischen Dimension und fragt nach der Praktikabilität in der Rechtswirklichkeit, um schließlich verfassungsrechtliche Aspekte, insbesondere den durch die Gesetzesbegründung vernachlässigten Grundsatz der Gerichtsöffentlichkeit, anzusprechen. Dabei wird herausgearbeitet, dass weder ein aktueller Regelungsbedarf vorliegt, noch die avisierte Gesetzesänderung tatsächlich im Stande ist, die intendierten positiven Folgen für den Gerichtsstandort Deutschland zu erreichen.

9 BR Drs. 42/10, S. 6 f.

10 BR Drs. 42/10, S. 6 f.

11 BR Drs. $42 / 10$, S. 7.

12 BR Drs. 42/10, S. 8.

13 Bundesrat, Plenarprotokoll der 866. Sitzung vom 12. Februar 2010, S. 11 ff.; Bundesrat, Plenarprotokoll der 869. Sitzung vom 7. Mai 2010, S. 118.

14 Handschell, Die Vereinbarkeit von Englisch als Gerichtssprache mit dem Grundgesetz und europäischem Recht, Deutsche Richterzeitung 2010, S. 395 ff.; Stellungnahme der Rechtsanwaltskammer Stuttgart, http://www.rak-stuttgart.de/index.php?id=1165 (abgerufen am 28.2.2011).

15 Calliess/Hoffmann, Effektive Dienstleistungen für den globalen Handel, Zeitschrift für Rechtspolitik 2009, S. 1ff.; Ewer, Das Öffentlichkeitsprinzip - ein Hindernis für die Zulassung von Englisch als konsensual-optionaler Gerichtssprache?, Neue Juristische Wochenschrift 2010, S. 1323 ff.; Remmert, Englisch als Gerichtssprache: Nothing ventured, nothing gained, ZIP - Zeitschrift für Wirtschaftsrecht 2010, S. 1579 ff.; Stellungnahme des Deutschen Anwaltvereins vom August 2010, http://anwaltverein.de/downloads/ stellungnahmen/SN-10/SN46.pdf (abgerufen am 2.1.2011).

16 Stubbe, Englisch als Gerichtssprache?, Zeitschrift für Rechtspolitik 2010, S. 195 f.; MaierReimer, Vertragssprache und Sprache des anwendbaren Rechts, Neue Juristische Wochenschrift 2010, 2545 ff.; Hilgard, Englisch als Gerichtssprache in Deutschland?, Betriebsberater 2010, S. 2969. 


\section{Bestehen eines Regelungsbedarfs: Das Argument vom Wettbewerb mit anderen Rechtsordnungen}

Zur Begründung des Gesetzentwurfs wird vorgetragen, dass es einen internationalen Wettbewerb der Gerichtsstandorte und Rechtssysteme gebe. ${ }^{17}$ Wenn dem tatsächlich so ist, würden andere Staaten ebenfalls die englische Sprache an ihren Gerichten zulassen bzw. würden ein solches Vorhaben zumindest planen, was - soweit ersichtlich - zumindest im europäischen Rechtskreis aktuell nicht der Fall ist. ${ }^{18}$ Die Voraussetzung der Entscheidung für oder gegen die Grundausrichtung des Gesetzesentwurfs ist es, zu wissen, mit welchen Staaten das deutsche Rechtssystem im Wettbewerb steht und gegebenenfalls welche Erfahrungswerte es mit der Einführung englischsprachiger Kammern in anderen Staaten gibt. Diesen zentralen Beleg bleibt die Gesetzesbegründung jedoch schuldig.

Es kann wohl davon ausgegangen werden, dass durch das Englische die Vergleiche - nicht die Vergleichbarkeit - zwischen den Rechtssystemen zunehmen. Das könnte dazu führen, dass weitere Angleichungen der Rechtsverfahren gefordert werden, da das englische Verfahrensrecht aus der (vom Common Law geprägten) internationalen Perspektive deutlich praktikabler ist. ${ }^{19}$ Davon würde das deutsche Zivilrecht nicht notwendigerweise profitieren. Im Gegenteil wäre sogar zu überlegen, ob es nicht gute Gründe gibt, den Bereich der Rechtsprechung vom Wettbewerb auszunehmen, um nicht wichtige Bereiche des gewachsenen Rechtsdiskurses und damit des effektiv-funktionierenden Rechtssystems zugunsten eines (behaupteten) ökonomischen Wettbewerbsvorteils zu opfern. Das Argument des $D A V$, Recht sei ähnlich wie Chemieindustrie oder Ingenieurswesen dem Wettbewerb anzupassen, ${ }^{20}$ zeugt von einem erschreckenden Rechtsverständnis.

Die Gründe im Gesetzentwurf gegen die Schiedsgerichte ${ }^{21}$ stehen im Widerspruch zur allseitigen Beliebtheit der Schiedsgerichtsbarkeit, die in der im Unterschied zu den staatlichen Gerichten vorhandenen Flexibilität liegt: Die Parteien können etwa das Beweisrecht beeinflussen, das Verfahren kann zudem vollständig in englischer Sprache durchgeführt werden. ${ }^{22}$ Bereits nach aktueller Rechtslage bietet das Schiedsgerichtsverfahren die Möglichkeiten, die durch den Gesetzentwurf gefordert werden, freilich ohne dabei das deutsche Recht notwendigerweise im Ausland populärer zu machen. Zwar ist der deutsche Standort in der Schiedsgerichtsbarkeit im Vergleich zu internationalen Schiedsgerichten durchaus konkurrenzfähig. ${ }^{23}$ Dies ist jedoch eher in der Effizienz der deutschen Schiedsrichter als in der Vorteilhaftigkeit des deutschen Rechts-

17 BR Drs. 42/10, S. 6.

18 Hoppe (Fn. 4), S. 376, der auf die bereits nach der geltenden Rechtslage bestehende Möglichkeit die Verhandlung auf Englisch zu führen, verweist, bezeichnet die Durchführung des gesamten Verfahrens, inklusive der Abfassung des Urteils in englischer Sprache, jedoch als beispiellos.

19 Wirth, Ihr Zeuge, Herr Rechtsanwalt! Weshalb Civil-Law-Schiedsrichter Common-LawVerfahrensrecht anwenden, Zeitschrift für Schiedsverfahren 2009, S. 9 ff.

20 Stellungnahme des Deutschen Anwaltvereins vom August 2010 (Fn. 15), Rn. 12.

21 BR Drs. 42/10, S. 7.

22 Vgl. § 1045 Abs. 1 BGB.

23 Berger, Herausforderungen für die (deutsche) Schiedsgerichtsbarkeit, Zeitschrift für Schiedsverfahren 2010, S. 291. 
systems begründet. An diesem Beispiel zeigt sich, dass es für den erfolgreichen Export des deutschen Rechts mehr bräuchte als die bloße Anpassung der Verfahrenssprache. ${ }^{24}$ Warum bei der Konkurrenzfähigkeit der deutschen Schiedsgerichte trotzdem die Idee verfolgt wird, den deutschen Gerichtsstandort zu internationalisieren, erschließt sich demgegenüber nicht.

\section{Zweckdienlichkeit der konkreten Änderungen}

In der Gesetzesbegründung wird argumentiert, das Gesetz helfe die Kongruenz von Vertrags- und Verfahrenssprache herzustellen und den „Sprachbruch“ zu vermeiden. ${ }^{25}$ Die Kongruenz von Vertrags- und Verfahrenssprache erleichtere wesentlich die Auslegung der Vertragstexte. ${ }^{26}$ Dagegen wird im Folgenden dargestellt, dass es gravierende Gründe gibt, die gegen eine Trennung von Rechts- und Verfahrenssprache sprechen, so dass im Ergebnis dem durch den Gesetzentwurf gewählten Ansatz der Auflösung dieser bisher untrennbaren Einheit mit Nachdruck zu widersprechen ist.

\section{Einheit von Rechtskultur und Sprache}

Die grundsätzliche Untrennbarkeit von Rechtskultur und Sprachkultur in Deutschland zeigt sich in mehreren Dimensionen. ${ }^{27}$ Die deutsche Sprache zählt als Staatssprache und als Umgangssprache zu den Verfassungsvoraussetzungen. ${ }^{28}$ Paul Kirchhof betont die „verfassungsnotwendige Mindestgemeinsamkeit der Sprache“. ${ }^{29}$ Aus diesem Grund ist es notwendig, erstens auf die Kontinuität der Rechtskultur in Beziehung zur Kontinuität der Sprache hinzuweisen: „Sprechweisen und Begriffe sind stark von der Tradition unseres Rechts, der Eigenart der Rechtskultur, den Funktionen der an der Rechtserzeugung und Rechtskonkretisierung beteiligten Organe und einer Dogmatik geprägt und gewinnen dadurch Bestimmtheit und Verläßlichkeit." ${ }^{30}$

Zweitens ist Recht das Medium des Demokratieprinzips, indem kommunikativ-gesellschaftliche Macht in administrative Macht umgesetzt wird, ${ }^{31}$ weshalb es sich nicht allein auf die Produzenten und Rezipienten der Staatsgewalten beschränken lässt. Das

24 Hilgard (Fn. 16).

25 BR Drs. 42/10, S. 7 u. S. 18.

26 BR Drs. 42/10, S. 6 f.

27 Die folgenden Aussagen beziehen sich sofern nicht anders vermerkt auf die verglichen mit z.B. Luxemburg oder der Schweiz sprachlich weitgehend homogene Bundesrepublik Deutschland.

28 Kirchhof, Die Identität der Verfassung in ihren unabänderlichen Inhalten, in: Isensee/ Kirchhof (Hrsg.), Handbuch des Staatsrechts Bd. I, 1987, § 19 Rn. 61.

29 Kirchhof, Mittel staatlichen Handelns, in: Isensee/Kirchhof (Hrsg.), Handbuch des Staatsrechts Bd. III, 1996, § 59 Rn. 174.

30 Kirchhof, Deutsche Sprache, in: Isensee/Kirchhof (Hrsg.), Handbuch des Staatsrechts Bd. I, 1987, § 18 Rn. 24; vgl. auch darstellend Schweizer, in: Veröffentlichungen der Vereinigung der Deutschen Staatsrechtslehrer, Bd. 65, 2006, S. 346 ff; Kahl, in: Veröffentlichungen der Vereinigung der Deutschen Staatsrechtslehrer, Bd. 65, 2006, S. 386 ff.

31 Habermas, Faktizität und Geltung, 1992, S. 23, S. 155, S. 182 ff. u. S. 428 f. 
Rechts ist nicht nur Fachsprache, ${ }^{32}$ es ist stets an die Gesamtheit adressiert: „Bei der verbindlichen Sprechweise, der Sprache des Rechts, muß der Staat allerdings stets die Sprache wählen, mit der er bei typisierender Betrachtungsweise alle Rechtsunterworfenen erreicht. Für die sprachhomogene Bundesrepublik Deutschland ist deshalb Deutsch die alleinige Rechtssprache." 33 Die faktische Kontinuität des Rechts in der deutschen Sprache wird von Kirchhof normativ gefasst: „Das Recht ist deshalb bereits durch seine sprachliche Verfasstheit gehalten, an Bestehendes anzuknüpfen, einen Änderungswillen auf Hergebrachtes zu beziehen, den Übergang so schonend zu gestalten, daß die Rechtsadressaten den Wechsel in ihrem Denken und damit in ihrer Sprache mitvollziehen können. “34

Die Verbindung von Rechtskultur und Sprache wird drittens unmittelbar einsichtig, wenn drei wesentliche Funktionen des Rechts betrachtet werden: Allein schon aufgrund des Integrationseffekts ist dem Staat daran gelegen, die gemeinsame Sprache in allen drei Gewalten zur Entfaltung zu bringen. Zweitens kann davon ausgegangen werden, dass es in „der Rechtsanwendung wesentlich verbesserte Wirkungsmöglichkeiten“ gibt, wenn Staat und Gesellschaft dieselbe Sprache teilen. ${ }^{35}$ Drittens liegen dem Rechtsstaatsprinzip die Überzeugungen zugrunde, zuvorderst sprachliche Kommunikation als Mittel der Ausübung staatlicher Aufgaben anzuwenden sowie Recht als positives Recht schriftlich zu fixieren bzw. zu verändern und dadurch berechenbar ${ }^{36} \mathrm{zu}$ machen, kurzum: „Rechtsstaatliche Gewalt ist eine Herrschaft des Wortes ${ }^{66^{37}}$ bzw.: „Rechtliches Tun ist sprachlich".38

Die folgenden Überlegungen gehen nicht von der absoluten Kohärenz von Rechtssprache und Rechtssystem aus; die Schweiz mag einmal mehr als Beispiel dienen, dass es einen derartigen ,organischen' Zusammenhang nicht gibt. Wohl aber gibt es gewachsene Rechtsdiskurse. Ferner wird im Gegensatz zu konservativen Vorstellungen nicht davon ausgegangen, dass die Sprachloyalität mit der politisch-staatlichen Loyalität gleichzusetzen ist.

a) Verbindung von Rechtssystem und Sprachkultur in der Rechtsprechung

Die oben erwähnten Kontinuitäten von Sprache und Recht treffen in der Rechtsprechung in dreifacher Weise aufeinander.

Erstens: Die allgemeinen Auslegungsmethoden der Rechtswissenschaft sind in hohem Maße Arbeit mit und an sprachlichen Zeichen: Die „Rechtsstaatlichkeit stützt sich auf die gemeinschaftliche Sprache“. ${ }^{39}$ Linguistisch betrachtet arbeitet die Rechtswissenschaft u.a. in den Kategorien der Wort- und Satzsemantik, der Grammatik, der Text-

32 Kirchhof (Fn. 30), Rn. 24.

33 Kirchhof (Fn. 30), Rn. 55; vgl. auch Rn. 45 ff.

34 Kirchhof (Fn. 30), Rn. 28.

35 Kirchhof (Fn. 30), Rn. 56.

36 Kirchhof (Fn. 30), Rn. 27.

37 Kirchhof (Fn. 30), Rn. 12.

38 Müller, Einige Grundfragen der Rechtslinguistik, in: Christensen/Müller (Hrsg.), Methodik, Theorie, Linguistik des Rechts, 1997, S. 64.

39 Kirchhof (Fn. 30), Rn. 2. 
und Diskurslinguistik, sie bedient sich des diachronen und synchronen sowie teilweise des inter-lingualen Vergleiches. Der Linguist Dietrich Busse hat nachgewiesen, dass es sich bei der Auslegung und Anwendung von Gesetzestexten nicht um das vermeintlich simple Verfahren der Subsumtion, sondern um die komplexe Tätigkeit des Wissens über zulässige Operation mit verschiedenen Textelementen handelt. ${ }^{40}$ Gerade weil es sich bei der Auslegung um das zentrale Arbeitsinstrument in der deutschen Rechtsanwendung handelt, wird auf sprachliche Genauigkeit in der juristischen Ausbildung wie auch in der täglichen Arbeit großen Wert gelegt. ${ }^{41}$ Um dieses Instrument anwenden zu können, d.h. frühere Entscheidungen zu verstehen und Gesetze nachzuvollziehen, muss ein hohes Maß an Sprachkompetenz vorhanden sein.

Zweitens: Sprachwandel und Sprachkontinuität sind zwei Sichtweisen auf dasselbe Phänomen; Sprache wandelt sich ständig, und doch erleben wir sie als weitgehend kontinuierlich. Die (juristische) Sprachkompetenz umfasst daher die Fähigkeit, Bedeutungswandel vor neuen sozialen und kulturellen Hintergründen wie auch den Bedeutungswandel innerhalb der juristischen Fachtexte bewerten zu können.

Drittens: Die Rechtsprechung ist weder empirisch noch normativ ein autonomer Teil des Rechtsdiskurses. Die Gewährleistung der Gesetzesbindung des Richters fordert das vollständige Gerichtsverfahren mit mündlichen und schriftlichen Aussagen aller Beteiligter. ${ }^{42}$ Das einzelne Gerichtsverfahren wird ferner vom nachfolgenden Instanzenzug, von der (Rechts)Wissenschaft und von der Öffentlichkeit kontrolliert: „Erst diese Faktoren zusammengenommen ergeben die Gesetzesbindung. Der Rechtsstaat ist nicht monologisch-richterbezogen, sondern diskursiv-verfahrensbezogen. Er verlässt sich nicht auf einsame Erkenntnis, sondern fordert eine öffentliche Diskussion, in der sich die besseren Argumente für die Lesart des Gesetzes durchsetzen."43 Der Rechtsstaat hat nicht nur eine Textstruktur, er ist eine Textstruktur. ${ }^{44}$ „Rechtsinstitute haben als Wissensrahmen damit im Wesentlichen eine Filter- und Ordnungsfunktion", sie institutionalisieren interpretationsrelevante Sinnzusammenhänge. ${ }^{45}$ Die Sicherung von Urteilen, Kommentaren und rechtswissenschaftlichen Texten dient der Etablierung eines Rahmens, der herrschende Meinungen und Interpretationen überhaupt erst konstant hält. ${ }^{46}$

40 Busse, Bedeutungsfeststellung, Interpretation, Arbeit mit Texten?, in: Haß-Zumkehr (Hrsg.), Sprache und Recht, 2002, S. 138 u. S. 160; Busse, Ist die Anwendung von Rechtstexten ein Fall von Kommunikation?, in: Lerch (Hrsg.), Die Sprache des Rechts Bd. III, 2005, S. 23.

41 Dass deutsche Juristen auch z.B. Englisch und Französisch lernen und sich mit dem USamerikanischen und französisches Rechtssystem auseinandersetzen, steht der rechtswissenschaftlichen Ausbildung für das deutsche Recht nicht entgegen.

42 Christensen/Sokolowski, Wie normativ ist Sprache?, in: Haß-Zumkehr (Hrsg.), Sprache und Recht, 2002, S. 64 ff.

43 Christensen/Sokolowski (Fn. 42), S. 74.

44 Müller/Christensen, Rechtstext und Textarbeit in der Strukturierenden Rechtslehre, in: Christensen/Müller (Hrsg.), Methodik, Theorie, Linguistik des Rechts, 1997, S. 85.

45 Busse, Ist die Anwendung von Rechtstexten ein Fall von Kommunikation? (Fn. 40), S. 39.

46 Busse, Ist die Anwendung von Rechtstexten ein Fall von Kommunikation? (Fn. 40), S. 40. 


\section{b) Allgemeine Übersetzungsschwierigkeiten}

Aus linguistischer Sicht liegt dem gesamten Gesetzentwurf ein problematisches Sprachverständnis zugrunde. ${ }^{47}$ Sprache wird hier ausschließlich als Werkzeug (in Anlehnung an Karl Bühler) betrachtet, welches je nach Zweck gewechselt werden kann, hier konkret in der Vorstellung der Übersetzung als Mittel zur Anwendbarkeit deutschen Rechts auf englische Texte. Heute ist man in Semiotik, Kommunikationswissenschaft und Linguistik der Auffassung, dass Sprache weniger ein Werkzeug denn (je nach Perspektive) ein sozial-kulturell gewachsenes wirklichkeitskonstituierendes Medium darstellt, welches Objekt- und Meta-Ebene in sich vereint. ${ }^{48}$ Diese Komplexität ignorierend wird in der Gesetzesbegründung die englische Sprache zur lingua franca im Sinne der genannten Organon-Vorstellung. Die für den (v.a. muttersprachlichen) kompetenten Sprecher täglich intuitiv benutzten vielfältigen Funktionen der Sprache (z.B. Synonymie und Vagheit, Zitation und Konnotation, Rückgriff auf Sprachgeschichte) werden ausgeblendet. Stattdessen entsteht mit dem Englischen eine vermeintlich pragmatische Arbeitssprache, deren Funktion sich im Ziel des Informationsaustausches zwischen Beteiligten nicht gleicher Sprache erschöpft. Die Einführung des Englischen für internationale Handelssachen erweist sich jedoch wegen der oben skizzierten Verbindung von Rechtsund Sprachkultur als wesentlich komplexerer.

Das Übersetzen juristischer Texte in eine Fremdsprache stellt sich nicht als die erhoffte pragmatische Lösung heraus, sondern führt gegenteilig zu neuen Problemen. Gerad-René de Groot mahnt: „Wesentlich ist, sich ständig bewusst zu machen, dass das Übersetzen juristischer Terminologie Rechtsvergleichung ist. “49 Daher ist zunächst zu fragen: Wie viele Rechtssysteme sind im Übersetzungsvorgang betroffen? Der Gesetzentwurf verfolgt zwar nicht das Ziel, eine in deutscher Sprache verfasste deutsche Rechtsnorm ins Englische derart zu übersetzten, dass die Übersetzung die im englischen Rechtssystem entsprechende Bezeichnung enthält. Stattdessen soll eine in deutscher Sprache verfasste deutsche Rechtsnorm in das Englische nach der Maßgabe übersetzt werden, dass die Übersetzung dem Verständnis des deutschen Rechtssystems weiterhin entspricht. Gleichwohl wird man um den Rechtsvergleich bei der onomasiologischen Suche nach begrifflichen Äquivalenzen nicht vollständig ausweichen können. Analog zu de Groot lassen sich theoretisch drei Wege für den Fall benennen, dass kein Äquivalent im Englischen gefunden wird: Nicht-Übersetzen des entsprechenden Ausdrucks (d.h. als markiertes Fremdwort belassen), Umschreiben (was dem Äquivalent am nächsten kommt und auch vom Gesetz angestrebt wird $)^{50}$ oder einen Neologismus entwickeln (dem Rechtszielsystem mittelbar einen neuen Ausdruck hinzufügen). ${ }^{51}$ Offensichtlich

47 Fehlende linguistische Kenntnis offenbart auch die Stellungnahme des Deutschen Anwaltvereins (Fn. 15), Rn. $31 \mathrm{f}$.

48 D.h. im Gegensatz zu anderen Kommunikationsmittel ist es möglich, mit Sprache über Sprache zu sprechen.

49 De Groot, Rechtsvergleichung als Kerntätigkeit bei der Übersetzung juristischer Terminologie, in: Haß-Zumkehr (Hrsg.), Sprache und Recht, 2002, S. 222; de Groot, Das Übersetzen juristischer Terminologie, in: de Groot/Schulze (Hrsg.), Recht und Übersetzen, S. $11 \mathrm{ff}$.

50 BR Drs. 42/10, S. 10.

51 De Groot, Rechtsvergleichung als Kerntätigkeit bei der Übersetzung juristischer Terminologie (Fn. 49), S. 232 ff.; de Groot, Das Übersetzen juristischer Terminologie (Fn. 49), S. 20 ff. 
bedarf es für die Übersetzung der zweisprachlichen und der juristischen Kompetenz in zwei Rechtssystemen.

Ein sprachliches Zeichen auf der Wort-Ebene hat zwei Seiten: eine Ausdrucks- und eine Inhaltsseite. ${ }^{52}$ Die Ausdrucksseite ist die Lautform eines Wortes in gesprochener und geschriebener Sprache, z.B. Hypothek ${ }^{53}$ Die Form allein stellt noch kein sprachliches Zeichen dar, denn mit ihr muss ein Inhalt verbunden sein, um das Zeichen als Sprachzeichen mit einer Bedeutung identifizieren zu können, hier ,Hypothek im Sinne des BGB'. Notwendige Voraussetzung für die Kommunikation mit sprachlichen Zeichen ist, dass die Zeichen innerhalb der Sprechergemeinschaft konventionell sind. Die Verknüpfung von Zeichenausdruck und Zeicheninhalt ist zwar wandelfähig (Sprachwandel, Bedeutungswandel), bedarf aber der weitgehend stabilen Konvention über die Zeichenbenutzung. Da zur eindeutigen Informationsvermittlung ein Zeichen in einem rechtlichen Verwendungskontext stets genau eine Bedeutung haben soll, gibt es im Rechtsdiskurs die die konventionelle Bedeutung näher bestimmenden entsprechenden Kommentare, wissenschaftlichen Hinweise und gerichtlichen Urteile. Das ist in der Fortentwicklung des Rechts vorgesehen und weitgehend unproblematisch. Problematisch wird es, wenn spontan entweder der Inhalt einen anderen Ausdruck erhält (Onomasiologie), also,Hypothek' plötzlich mortgage heißt, oder der Inhalt eines Ausdrucks sich ändert (Semasiologie). Onomasiologisch gilt wiederum zu unterscheiden, dass es möglich ist, dass der Inhalt in einem Zeichenausdruck sich niederschlägt, der nicht existiert, z.B. die Übersetzung von Hypothek als Neologismus hypothec. ${ }^{54}$ Ausgeschlossen ist selbstverständlich die semasiologische Möglichkeit der Beibehaltung des Ausdrucks bei wechselndem Inhalt, da das Ziel gerade im Austausch der deutschen durch englische Rechtsbegriffe besteht. Grundsätzlich scheint im Gesetzesentwurf die Vorstellung zu herrschen, dass die in der deutschen Rechtssystematik ruhende Bedeutung beibehalten bleibt, wenn der deutsche Ausdruck durch einen englischen Ausdruck ersetzt wird, also der Inhalt eines deutschen Ausdrucks fortan auch vom gewählten Ausdruck des englischen Zeichensystems repräsentiert wird. Trotz des dabei notwendigerweise impliziten Rechtsvergleiches wird eigentümlicherweise übersehen, dass der gewählte englische Ausdruck keineswegs ,semantisch leer' ist. Um ihn als sprachliches Zeichen nutzen zu können, muss dieser bereits über einen eigenen Zeicheninhalt verfügen, d.h. eine Bedeutung im Englischen ${ }^{55}$ besitzen: mortgage hat bereits eine Bedeutung im Common-Law und wird diese auch behalten. Damit werden die Rezipienten mit der Ambiguität des Zeichenausdrucks konfrontiert, dessen Bedeutung einerseits im englischen und andererseits im deutschen Rechts- bzw. Sprachsystem liegt. Als praktisches Beispiel kann hier die unterschiedliche Bedeutung des Wortes Vertrag (con-

52 Zum Folgenden de Saussure, Grundlagen der Allgemeinen Sprachwissenschaft, Hrsg. von Bally/Sechehaye, 1967, S. 76 ff.

53 Vgl. de Groot, Rechtsvergleichung als Kerntätigkeit bei der Übersetzung juristischer Terminologie (Fn. 49), S. 235; vgl. Triebel, Pitfalls of English as a Contract Language, in: Olsen/ Lorz/Stein (Hrsg.), Translation Issues in Language and Law, 2009, S. 150.

54 Die Erfindung von Neologismen für alle Nicht-Äquivalenzen verstärkt lediglich die Trennung vom deutschsprachigen und englischsprachigen Rechtsdiskurs.

55 Dies stellt auch Maier-Reimer fest (Fn. 16), S. 2547, nur unterscheidet er zwischen ,reinem Sprachsinn' und ,rechtlich geprägtem Sinn' von Texten, was im Fall der Rezeption als Auslegung nicht zu trennen ist. 
tract) im deutschen Recht und im Common-Law angeführt werden. Was im CommonLaw als contract bezeichnet wird, wird im Deutschen als vertragliches Schuldverhältnis charakterisiert. So bezeichnet das Wort Vertrag im Deutschen lediglich die Vereinbarung selbst, ${ }^{56}$ während der englische Begriff contract sämtliche aus der Vereinbarung entstehenden Rechte und Pflichten umfasst. ${ }^{57}$ Zahlreiche englische Rechtsbegriffe und Konzepte können zudem nur vor dem Hintergrund des Common-Law gesehen werden und haben schließlich oftmals kein Äquivalent im kontinentaleuropäischen Recht, wie beispielsweise die grundlegende Bedingung für das Zustandekommen eines Vertrages nach englischem Recht, die ,consideration'. ${ }^{58}$ Weiterhin haben zahlreiche englische Rechtsbegriffe eine prozessuale Bedeutung, während das deutsche Äquivalent dem materiellen Recht zuzuordnen ist, was zusätzlich Schwierigkeiten in der Rechtsanwendung bürgt. ${ }^{59}$ Das Rechts-Englisch ist daher insgesamt nicht vom Common-Law trennbar. ${ }^{60}$ Das Problem der semantischen Vagheit könnte sich zudem als iterativ herausstellen, weil im Gesetzentwurf von „Englisch“ die Rede ist, womit nicht eindeutig bestimmt ist, ob die juristischen Begriffe der Fachsprache des Commonwealth- oder des US-amerikanischen Rechtssystems gemeint sind. ${ }^{61}$ Ursache für die Inkohärenz der Begriffe im englischsprachigen und deutschen Rechtsdiskurs wären vor allem die jeweilige Ausbildung der textproduzierenden und -rezipierenden Richter. ${ }^{62}$ In jedem Fall wäre es ratsam, hinter der Übersetzung den deutschen Begriff als Fremdwort im englischen Text zu vermerken. ${ }^{63}$

Nicht nur die korrekte Übersetzung der englischen Fachsprache, sondern auch die Übersetzung von nicht fachlich geprägten Begriffen kann bisweilen erhebliche Schwierigkeiten bereiten. ${ }^{64}$ Bekanntlich sind selbst Gerichte mit muttersprachlichem Personal bei diesen Aufgaben stark gefordert. War bisher nur die Rede von morphologischer Ambiguität juristischer Fachbegriffe, so verschärft die adäquate Überführung syntaktischer, phraseologischer und stilistischer Eigenheiten der deutschen juristischen Fachsprache die Translation ins Englische. Im Übersetzungsvorgang erweist sich oftmals der treffende Gebrauch der Präpositionen sowie ihre Verbindung mit den Verben in der englischen Sprache als Fehlerquelle; ein Blick in die Literatur zur Praxis des Unterrichtens und Urteilens weist zudem neben der Lexik auch Modalverben als Quelle von

56 Tilch/Arloth, Deutsches Rechtslexikon, 2001, S. 5476.

57 Markesinis/Unberath/Johnston, The German Law of Contract - A Comparative Treatise, 2006, S. 25.

58 Hürten, Das Erfordernis der Gegenleistung (consideration) im englischen Vertragsrecht, 2004, S. $78 \mathrm{ff}$.

59 Beispiele bei Triebel (Fn. 53), S. 154 ff.

60 Triebel (Fn. 53), S. 149.

61 Als Grund für die Auseinanderentwicklung des englischen und des US-amerikanischen Rechts wird vielfach der Einfluss des europäischen Gesetzgebers angeführt, vgl. Markesinis/ Unberath/Johnston (Fn. 57), S. 2; als Beleg für die unangemessene Thematisierung der Sprachwahl dient neben der Gesetzesvorlage selbst auch die Diskussion, z.B. bei Calliess/ Hoffmann (Fn. 15).

62 Stubbe (Fn. 16), S. 196 warnt sogar, die Regiolekte könnten zu Verständnisschwierigkeiten führen.

63 So auch der Hinweis von Maier-Reimer (Fn. 17), S. 2550.

64 Triebel (Fn. 53), S. 154. 
Übersetzungsschwierigkeiten aus ${ }^{65}$ sowie Konjunktionaladverbien und Konjunktionen, welche nicht nur mitzuübersetzende Bindungsglieder sind, sondern in juristischen Texten großer Aufmerksamkeit bedürfen. ${ }^{66}$

\section{c) Konventionalisierung und Rechtsbindung}

Die Vorstellung des durch den Richter allein sprechenden Gesetzgebers resultiert aus dem verfassungsrechtlichen Gebot der Gewaltenteilung und begründet sich in der Überzeugung, dass die Norm und damit auch die Lösung bereits im Gesetzestext enthalten sind. ${ }^{67}$ Dass eine solche Vorstellung heute nicht mehr haltbar ist, zeigen Rechtspraxis und rechtslinguistische Forschungen. ${ }^{68}$ Bekanntlich arbeitet der Jurist nicht nur mit Begriffen, sondern insbesondere an Begriffen. ${ }^{69}$ Rechtsanwendung heißt daher Handeln im Rahmen einer inter-textuellen bzw. diskursiv geprägten Auslegungspraxis. Die Bindung des Juristen an das Recht erfolgt in der konkreten Arbeit mit den Rechtstexten, den Urteilen und den Kommentaren. ${ }^{70}$ Müller/Christensen/Sokolowski sprechen daher zurecht vom „semantischen Kampf" und vom ,juristischen Handeln als semantischer Praxis““.$^{71}$ Der Verbindlichkeitsanspruch in der praktischen Rechtsarbeit, eine Regel für eine Bedeutung zu bestimmen, ist dabei ein Akt der Sprachnormierung. ${ }^{72}$ Der Gesetzestext wird hinc et nunc durch den Richter produziert, indem aus dem vorliegenden Fall ein rechtlicher Fall wird. Die aufeinander aufbauenden Sprachnormierungsakte ergeben das Rechtssystem, welches wiederum in Maßen auf den Normierungsakt eingeht. Die Anwendung des Rechts ist Auslegung und zugleich performative Produktion von neuen Texten. Es ist gerade das vor allem aus der deutschen Sprache bestehende diskursive Kontinuum, das durch den Wechsel ins Englische gefährdet wird. Denn beim Übersetzen durch den Richter kommt diesem ein noch nie dagewesener Spielraum in der Urteilsfindung zu, dessen Kontrolle sich früher aus der Rechtsauslegung ergab, die, wenn auch nicht kodifiziert, so doch systematisiert war. Demgegenüber stehen nunmehr ad hoc-Übersetzungen, die zu Okkasionalismen führen, ohne notwendigerweise einer strukturierenden Rechtsauslegung zu entsprechen. Der aus dem Gewaltenteilungsprinzip resultierende Schutz vor richterlicher Willkür könnte dadurch eingeschränkt werden. Diese schwindende Bindung des Richters an das Recht vollzieht sich zugleich durch die vermutlich eingeschränkte Rezeption der englischsprachigen Urteilsbegriffe: Die „Zitathaftigkeit“, so Derrida, ist eine notwendige Eigenschaft eines jeden Zeichens, um als

65 Hier Französisch-Deutsch, vgl. Ballansat/Perrin, Kombinierte Analyse von Inhalt und Sprache juristischer Texte als Vorbereitung auf den Übersetzungsunterricht, in: Heller/Ehlich (Hrsg.), Studien zur Rechtskommunikation, 2007, S. 275 ff.

66 Pescatore, Das Konzipieren übersetzungsgerechter juristischer Dokumente, in: de Groot/ Schulze (Hrsg.) (Fn. 49), S. 95.

67 Müller (Fn. 38), S. 58 f.

68 Müller/Christensen (Fn. 44); Busse (Fn. 40); Müller/Christensen/Sokolowski, Rechtsarbeit und Textarbeit, 1997.

69 Müller (Fn. 38), S. 61.

70 Müller/Christensen, Rechtstext und Textarbeit in der Strukturierenden Rechtslehre, in: Christensen/Müller (Fn. 39), S. 86 ff.

71 Müller/Christensen/Sokolowski (Fn. 68), S. 59 u. S. 72.

72 Müller/Christensen/Sokolowski (Fn. 68), S. 73. 
Zeichen erkannt zu werden. ${ }^{73}$ Die Ersetzung eines deutschen Ausdrucks durch einen englischen Begriff muss demnach von anderen Juristen verstanden und in irgendeiner Art übernommen werden, um sie zu Zeichen innerhalb des Rechtssystems werden zu lassen. Andernfalls bleiben die Begriffe in den Urteilen unsystematisierbare Erscheinungen. Es steht allerdings zu befürchten, dass das englischsprachige Urteil nicht ohne weiteres zur Grundlage weiterer juristischer Textarbeit werden kann, weil die Okkasionalismen ohne ihre Genese vermutlich kaum verstanden werden.

\section{d) Zwischenergebnis}

Zusammenfassend erscheinen mindestens drei Punkte fragwürdig: Das Verfahren in englischer Sprache wird die sprachlich vollzogenen Akte der Interpretation des Parteiwillens und der Subsumtion unter deutsches Recht lediglich hinauszögern, obwohl es doch zu vermuten steht, dass die Verständigung über den Textinhalt den Kern des Rechtsstreits darstellt. ${ }^{74}$ Zweitens: In Folge der Arbeit der geforderten Kammern wird sich innerhalb der deutschen Rechtskultur ein englischsprachiger Diskurs entwickeln, der in seiner Erscheinung ausdrucksseitig anschlussfähig an das englische Rechtssystem scheint, mit diesem inhaltlich aber nicht übereinstimmt. Weil es sich aber um englischsprachige Texte handelt, wird das Subsystem auf der anderen Seite an den deutschen Rechtsdiskurs kaum ohne weiteres anschlussfähig sein. Der Sprachbruch wird im Rechtsdiskurs tradiert. Drittens: Die hiesige Rechtsdogmatik wird durch die Vermischung bzw. den englischsprachigen Paralleldiskurs nicht eben an Schärfe zunehmen. Wenn das deutsche Zivilrecht so gut und eigentlich auch begehrt ist, wie behauptet, ${ }^{75}$ warum es dann ausgerechnet der Gefahr aussetzen, es zu verschlechtern? Oder: Warum das mit der Sprache gewachsene Instrument des Rechts zugunsten des (angenommenen) Wettbewerbs aus der Hand geben?

\section{Intendierte Verfahrensvereinfachung}

Weiterhin muss auch in Frage gestellt werden, ob die durch den Gesetzesentwurf intendierte Verfahrensvereinfachung tatsächlich erreicht wird. Zunächst muss darauf verwiesen werden, dass in den Verfahren vor dem Bundesgerichtshof weiterhin grundsätzlich die deutsche Sprache gilt, lediglich fakultativ kann das Verfahren auch auf Englisch fortgesetzt werden. ${ }^{76}$ Für die Anfangsphase ist jedoch kaum damit zu rechnen, dass auch beim Bundesgerichtshof Richter zur Verfügung stehen, die ein Verfahren ohne Qualitätseinbußen vollständig in englischer Sprache durchführen können. ${ }^{77}$ Würde das Verfahren aber auf Deutsch fortgesetzt werden, so müsste das erstinstanzliche Urteil übersetzt werden, was aufgrund der dargestellten Schwierigkeit, englischsprachige

73 Derrida, Signatur Ereignis Kontext, in: Derrida, Randgänge der Philosophie, 1988, S. 339.

74 Vgl. Maier-Reimer (Fn. 16), S. 2550.

75 Broschüre Law Made in Germany (2008), http://www.lawmadeingermany.de/Law-Made_in_Germany.pdf (abgerufen am 2.1.2011).

76 BR Drs. 42/10, S. 3.

77 Vgl. auch Hoppe (Fn. 4), S. 376. 
Rechtstexte an den deutschen Rechtsdiskurs anzuschließen, auf Rechtsanwendungsebene erhebliche Fehlerquellen birgt. ${ }^{78}$ Die Überprüfung der Rechtsanwendung durch die Vorinstanzen und die Einordnung in die bisherige Rechtsprechung ist jedoch ureigene Aufgabe des Revisionsverfahrens, ${ }^{79}$ welche durch die dargestellten Übersetzungsschwierigkeiten erheblich beeinträchtigt werden würde.

\section{Steigerung der Popularität des Gerichtsstandorts und des deutschen Rechts?}

Der Gesetzentwurf verfolgt eine doppelte Zielsetzung: Der Gerichtsstandort Deutschland soll einerseits insgesamt attraktiver gemacht werden, andererseits soll auch das deutsche Recht auf internationale Verträge vermehrt Anwendung finden. ${ }^{80}$ Es ist jedoch nicht ersichtlich, wie die in dem Gesetzentwurf vorgeschlagenen Regelungen tatsächlich die Wettbewerbsfähigkeit der deutschen Rechtsordnung fördern können, d.h. für seine Vorzüge zu werben imstande ist. ${ }^{81}$ Die politische Überzeugung, nach der Deutschland über das beste Recht verfügt und dieses verbreitet werden soll, wie etwa die Initiative „Law made in Germany“ postuliert, ${ }^{82}$ ist dabei von der Forcierung des zwischenstaatlichen Wettbewerbs um beträchtliche finanzielle Streitigkeiten zu trennen. ${ }^{83}$ Letzteres Ziel betrifft nämlich den primär monetären Faktor, durch Anziehung von Streitigkeiten mit hohen Streitwerten der Staatskasse gleichzeitig hohe Gerichtsgebühren zuzuführen. Ein Export des deutschen Rechts kann mit der Einführung der englischen Sprache an deutschen Zivilgerichten bereits deshalb nicht verbunden sein, da es sich bei dem durch den Gesetzesentwurf gewählten Mechanismus primär nicht um einen Rechtsexport, sondern um einen Import von Rechtsstreitigkeiten und Rechtsunterworfenen handelt. Ob dies zwangsläufig zu einem Export des deutschen Rechts führen kann, muss, auch vor dem Hintergrund der mangelnden Regelung über eine standardisierte Übersetzung deutscher Rechtsquellen, ${ }^{84}$ bezweifelt werden. Es bestehen neben der Sprache weitere durchaus gewichtige Hindernisse, die der Wahl des deutschen Rechts in ausländischen Streitigkeiten entgegenstehen. Es erscheint zunächst unerlässlich, dass jeweils ein im deutschen Recht ausgebildeter Jurist an dem Prozess beteiligt ist, was den Vorteil der Verfahrenssprache nivelliert. ${ }^{85}$ Das deutsche Zivilprozessrecht weist, insbesondere im Hinblick auf das Beweisreicht, gegenüber den Verfahrensrechten des Common Law zahlreiche Besonderheiten auf. ${ }^{86}$ Es erscheint insofern schwer vorstellbar, dass sich ein im Common Law ausgebildeter Jurist im deutschen Zivilprozessrecht ebenso zurecht-

78 Hoppe (Fn. 4), S. 376.

79 Rimmelspacher, Münchener Kommentar zur Zivilprozessordnung 2007, Vorbemerkung zu den $\S \S 511$ ff. Rn. 6.

80 BR Drs. 42/10, S. 8.

81 Vgl. auch Hilgard (Fn. 16).

82 Broschüre Law Made in Germany (Fn. 75).

83 So argumentieren Calliess/Hoffmann (Fn. 15) mit Vorteilen für die auf den Export angewiesenen deutschen Wirtschaft für ,eine Investition in effektive Justizdienstleistungen als spezielle Form von Standortpolitik“".

84 Weiteres dazu siehe unten.

85 So auch Hoppe (Fn. 4), S. 375.

86 Reitz, Grundlegende Unterschiede zwischen dem deutschen und dem U.S.-amerikanischen Zivilprozess: Vorzüge, die sich ausschließen?, Zeitschrift für Zivilprozess 1991, S. 381 ff. 
findet wie ein deutscher Jurist und damit prozessuale Waffengleichheit hergestellt werden kann. Dann ist es aber auch nicht ersichtlich, warum ein internationaler Handelspartner der Wahl des deutschen Gerichtsstandortes zustimmen sollte. Das deutsche materielle Zivilrecht ist zudem inhaltlich in vielerlei Hinsicht aus internationalem Blickwinkel nicht „,konkurrenzfähig“, was das Beispiel des AGB-Rechts verdeutlicht. ${ }^{87}$ Die aufgezeigten Hindernisse lassen das Ziel, das deutsche Recht international größerer Geltung zu verschaffen, lediglich als eine diffuse Hoffnung erscheinen.

\section{Ausgestaltungsdefizite}

Der Gesetzesentwurf lässt bezüglich der konkreten Umsetzung der Übersetzungsleistung viele Fragen ungeklärt. Er verhält sich insbesondere zu der Frage, wie die Übersetzung bzw. Umschreibung ${ }^{88}$ deutscher Rechtsbegriffe ins Englische praktisch erfolgen soll, in keiner Weise. Die sich aufdrängende Frage nach einer Standardisierung englischer Übersetzungen von deutschen Rechtsbegriffen bleibt völlig offen. Es böte sich geradezu an, über eine verbindliche Übersetzung von ZPO und BGB nachzudenken, um zumindest die einheitliche Verwendung englischer Begriffe für die verschiedenen Rechtsinstitute zu gewährleisten, was offensichtlich nicht geschehen ist und was die Holzschnittartigkeit des gesamten Gesetzesentwurfs verdeutlicht. Inspirierend könnten die „Pinyin“-Kommission in den Niederlanden sein, die Vorschläge für Standardübersetzung von niederländischen Begriffen ins Deutsche, Englische und Französische vorschlägt, ${ }^{89}$ sowie das für einfache Übersetzungen Deutsch-Englisch und vice versa eintretende Plain Language Movement. ${ }^{90}$ Konsequenterweise und vor dem Hintergrund des Gesetzesentwurfs, für internationale Rechtsstreitigkeiten eine attraktive Alternative zu Schiedsgerichtsverfahren anzubieten, müssten alle erdenklichen Rechtsquellen ins Englisch übersetzt werden. Nur dann könnte auch ein formal gleichwertiges Agieren der nicht muttersprachlichen Parteien gewährleistet werden.

Als Unterstützung für die Kammern für internationale Handelssachen, um die weitaus schwierigere semantisch-funktionale Frage zum Verhältnis von englischsprachigen deutschen und deutsch-deutschen Textelementen zu bewältigen, sieht der Gesetzesentwurf lediglich Personalentwicklungsmaßnahmen in Form von Fortbildungen vor. ${ }^{91}$ Diese müssen notwendigerweise umfassend sein, nimmt man die Ausbildung von Übersetzern, denen neben den Fremdsprachen auch Fachwissen und linguistisches Wissen vermittelt wird, als Maßstab. ${ }^{92}$ Jedoch ist treffendes, strukturiertes und nachhaltiges Übersetzen von Fachtexten teuer. Diese immensen Fortbildungskosten für Richter berücksichtigt der Gesetzentwurf nicht. Dadurch wird die vermeintliche Kosteneffizienz der deutschen Zivilgerichte um diese nicht externalisierbaren Kosten vermindert. Die Erarbeitung standardisierter Übersetzungen und die Einstellung von ausgebildeten

87 Stubbe (Fn. 16), S. 196; Hilgard (Fn. 17).

88 BR Drs. 42/10, S. 9.

89 Georgen/Schulze (Fn. 2), S. 42 f.

90 Hammel, Legal Translation, the Plain Language Movement, and English as a Lingua Franca, in: Olsen/Lorz/Stein (Hrsg.), Law and Language, 2008, S. 267 ff.

91 BR Drs. 42/10, S. 8 f.

92 Ballansat/Perrin (Fn. 65). 
Übersetzern für Deutsch-Englisch sind ebenfalls Kosten, die der Gesetzentwurf nicht berücksichtigt. Kostengünstige und qualitativ hochwertige Alternativen wie Wörterbücher $^{93}$ vermögen dies nicht zu leisten.

\section{Verfassungsrechtliche Implikationen}

Durch die teilweise Umstellung der Verfahrenssprache auf Englisch wird der Öffentlichkeitsgrundsatz tangiert. ${ }^{94}$ Dieser wird aus dem Rechtsstaatsprinzip ${ }^{95}$ hergeleitet und ist Bestandteil des Öffentlichkeitsprinzips der Demokratie. ${ }^{96}$ In seiner rechtsstaatlichen Komponente zielt er darauf ab, die Einhaltung des formellen und materiellen Rechts zu gewährleisten und zu diesem Zweck Einblicke in die Funktionsweise der Rechtsordnung zu ermöglichen. ${ }^{97}$

\section{Umfang der Gewährleistung}

Der Grundsatz der Gerichtsöffentlichkeit beschränkt sich in seinem Geltungsbereich von vornherein auf die mündliche Verhandlung. ${ }^{98}$ Rein schriftliche Verfahren, etwa nach $\S 128$ Abs. 2 ZPO oder nach $\S 101$ Abs. 2 VwGO tangieren diesen Grundsatz daher nicht. Dabei ist davon auszugehen, dass gerade die Anwesenheit sämtlicher an dem Verfahren Beteiligter eine weitaus größere Gefahr für das Ziel der Transparenz des Verfahrens darstellt, als dies bei schriftlichen Verfahren der Fall ist, was die Beschränkung der Gewährleistung von vornherein auf die mündliche Verhandlung rechtfertigt.

\section{Beschränkung des Öffentlichkeitsgrundsatzes}

Aus dem Grundsatz der Gerichtsöffentlichkeit kann grundsätzlich kein Anspruch des Einzelnen auf Verstehen des Verfahrens abgeleitet werden ${ }^{99}$ - es besteht insoweit kein subjektives Recht auf umfassendes Miterleben der Gerichtsverhandlung. ${ }^{100}$ Der Grundsatz wird jedoch in seinem objektiven Gehalt tangiert, indem der Öffentlichkeit insgesamt das Verfolgen der Gerichtsverhandlung erschwert wird. Zur genaueren Umgrenzung und Charakterisierung des Grundsatzes wird teilweise darauf abgestellt, dass primär verhindert werden soll, dass sich die Parteien unbeobachtet fühlen, auf ein tatsäch-

93 De Groot, Rechtsvergleichung als Kerntätigkeit bei der Übersetzung juristischer Terminologie (Fn. 49), S. $236 \mathrm{f}$.

94 BR Drs. 42/10, S. 8 f.

95 BVerfGE 103, 44 (63) und (75).

96 BVerfGE 70, 324 (358); Degenhart, Gerichtsverfahren, in: Isensee/Kirchhof (Hrsg.), Handbuch des Staatsrechts Bd. III, 1996, § 76 Rn. 50.

97 BVerfGE 103, 44 (65).

98 BVerfGE 103, 44 (63).

99 Kissel/Meyer, Gerichtsverfassungsgesetz: Kommentar, 2008, § 169 Rn. 52.

100 Von Coelln, Zur Medienöffentlichkeit der Dritten Gewalt, 2005, S. 121. 
liches Verständnis soll es dabei nicht ankommen. ${ }^{101}$ Beschränkt sich der Grundsatz der Gerichtsöffentlichkeit danach lediglich darauf, dass irgendwie geartete Vorkehrungen gegen die Heimlichkeit getroffen werden, so müsste man dogmatisch sauber bereits die Beschränkung des Öffentlichkeitsgrundsatzes durch den Wechsel der Gerichtssprache verneinen. Die abstrakte Kontrollmöglichkeit bleibt nämlich insofern erhalten.

Auf der anderen Seite ist zu bedenken, dass ein Verständnis, welches den Grundsatz der Gerichtsöffentlichkeit auf eine bloß symbolische Kontrolle beschränkt, im Ergebnis zu kurz greift. Denn die Möglichkeit der öffentlichen Kontrolle darf nicht zu einer bloß abstrakten, rein formalen Kontrollmöglichkeit werden. Zur Verdeutlichung kann die Alternative ins Feld geführt werden, Chinesisch als Gerichtssprache einzuführen. Die Möglichkeit der Wahl dieser deutlich geringer verbreiteten Sprache als dem Englischen würde, was wohl allgemein anerkannt sein dürfte, die bloß potenziell geforderte Kontrollmöglichkeit des Publikums unterminieren und den Öffentlichkeitsgrundsatz zu einer inhaltsleeren Formalie verkommen lassen. Insofern liegt dem Gesetzentwurf letztlich doch die implizite Annahme zugrunde, dass Englisch verstanden wird und es somit doch nicht allein um potenziell verstehende, sondern um tatsächlich verstehende Anwesende geht. ${ }^{102}$ Es kann aber gerade nicht davon ausgegangen werden, dass das Vorhandensein einer gewissen Kompetenz in englischer Umgangssprache in Teilen der deutschen Gesellschaft ${ }^{103}$ das Verfolgen einer Gerichtsverhandlung auf Englisch ermöglicht. ${ }^{104}$ Dies wird allein Juristen mit sehr guten Kenntnissen in der englischen Fachsprache möglich sein, wie die Begründung des Gesetzentwurfs mit Verweis auf die Zusatzqualifikation des richterlichen und nichtrichterlichen Personals selbst in Teilen einräumt. ${ }^{105}$ Die Wahl von Englisch als Verfahrenssprache stellt im Ergebnis eine Einschränkung des Öffentlichkeitsgrundsatzes dar.

\section{Rechtfertigung der Einschränkung durch gegenläufige Verfassungsgüter}

Liegt eine Einschränkung eines verfassungsrechtlichen Grundsatzes bzw. einer verfassungsrechtlichen Garantie vor, so muss diese auch auf Verfassungsebene gerechtfertigt werden. ${ }^{106}$ Eine Einschränkung des Öffentlichkeitsgrundsatzes kann insofern durch entgegenstehende Belange des Allgemeinwohls gerechtfertigt werden, soweit dies von

101 Müller-Piepenkötter (Fn. 5), S. 5; Bockelmann, Öffentlichkeit und Strafrechtspflege, Neue Juristische Wochenschrift 1960, 218.

102 So im Ergebnis auch Handschell (Fn. 14), S. 396.

103 BR Drs. 42/10, S. 12.

104 Zutreffender als die in der Gesetzesbegründung zitierte Umfrage zur Englischkompetenz der Deutschen ist das Ergebnis einer ebenfalls von der GfdS durchgeführte Studie: In Zusammenarbeit mit dem Zentrum für Rechtslinguistik und dem IfD Allensbach wurde nachgewiesen, dass 86 Prozent der Befragten Schwierigkeiten haben, Schreiben von Behörden, Anwälten etc. zu verstehen. Bereits jetzt herrscht die umfassende Tendenz zur Verselbstständigung der Rechtssprache, die sich dadurch von der Alltagssprache abhebt, vgl. Kirchhof, Rechtsprechen ist mehr als Nachsprechen von Vorgeschriebenem, in: Haß-Zumkehr (Hrsg.), Sprache und Recht, 2002, S.122.

105 BR Drs. 42/10, S. 14.

106 BVerfGE 103, 44 (75). 
Verfassungswegen geboten ist. ${ }^{107}$ Das Bundesverfassungsgericht führt als Rechtsgüter, zugunsten derer die Beschränkung des Öffentlichkeitsgrundsatzes gerechtfertigt werden kann, das Persönlichkeitsrecht, den Anspruch auf ein faires Verfahren, die Funktionstüchtigkeit der Rechtspflege sowie die ungestörte Wahrheits- und Rechtsfindung an. ${ }^{108}$ Sämtliche Güter sind durch die einfach-gesetzliche Ausgestaltung des Öffentlichkeitsgrundsatzes im GVG umgesetzt worden. ${ }^{109}$ Die Gesetzesbegründung thematisiert das Vorliegen eines solchen gegenläufigen Verfassungsgutes jedoch in keiner Weise. Eine verfassungsrechtliche Rechtfertigung scheint auch nicht offenkundig vorzuliegen: Dass die Wahl der Gerichtssprache der Vereinfachung des Verfahrens dient, darf jedenfalls bezweifelt werden. Ob die weiteren für die Notwendigkeit der Gesetzesänderung angeführten Gründe ausreichen, um die Einschränkung der Gerichtsöffentlichkeit zu rechtfertigen, ist zumindest zweifelhaft. Zwar hat der Gesetzgeber einen gewissen Spielraum bei der Ausgestaltung des Öffentlichkeitsgrundsatzes, ${ }^{110}$ allerdings ist eine Abwägung der gegenläufigen Verfassungsgüter erforderlich. Diesbezüglich muss festgehalten werden, dass weder die Verbreitung des deutschen Rechts im Ausland noch die Stärkung der Wettbewerbsfähigkeit deutscher Unternehmen im internationalen Wettbewerb schlechthin durch die Verfassung geschützte Interessen darstellen. Gleiches gilt auch für das Interesse daran, internationale Rechtsstreitigkeiten den deutschen Zivilgerichten zuzuführen. Soweit argumentiert wird, dass, wenn dem Gesetzgeber die Beschränkung der Öffentlichkeit in Disziplinarverfahren nicht verwehrt ist, a majore ad minus die Einschränkung des Öffentlichkeitsgrundsatzes in zivilrechtlichen Verfahren, die der Dispositionsbefugnis der Parteien unterliegen, erlaubt sein muss, ${ }^{111}$ so verkennt dies grundlegend die Notwendigkeit einer verfassungsrechtlichen Rechtfertigung. Die Einschränkung der Öffentlichkeit in Disziplinarverfahren ist demgegenüber ausdrücklich durch den Schutz des Betroffenen gerechtfertigt. ${ }^{112}$

\section{Fazit}

Die herausragende Rolle der Sprache in der Rechtswissenschaft, der Verbindung von Sprachsystem und Rechtssystem sowie des Rechtsstaats als Diskurs bildete bislang einen Konsens, der nicht infrage gestellt wurde. Weil Deutsch als Gerichtssprache im Gerichtsverfassungsgesetz unmissverständlich definiert ist, wurde sie beispielsweise von Kirchhof kaum problematisiert. ${ }^{113}$ Dies ändert sich mit dem hier diskutierten Gesetzesvorhaben, das den Konsens auflöst.

Aus linguistischer Sicht kann prognostiziert werden, dass mit der Einführung des Englischen als Hilfssprache eine dritte Rechtsterminologie neben der deutschen juristischen Fachsprache des deutschen Rechtssystems und der juristischen Fachsprache der

107 BVerfGE 70, 323 (358); 103, 44 (64); Handschell (Fn. 14), S. 398.

108 BVerfGE 103, 44 (63).

109 BVerfGE 103, 44 (64).

110 BVerfGE 103, 44 (64).

111 Ewer (Fn. 15), S. 1325.

112 BVerfGE 4, 74 (94).

113 Kirchhof (Fn. 30), Rn. 48. 
englischsprachigen Rechtssysteme entstehen wird, die eine eigene Semantik und damit zwangsläufig auch ein eigenes Auslegungssystem ausbilden wird.

Die oben dargestellten Hindernisse und Gefahren für die deutsche Rechtskultur und den deutschsprachigen Rechtsdiskurs wiegen aus unserer Sicht so schwer, dass die vermeintlichen Vorteile der Verfahrenssprache Englisch diese kaum überwiegen. Schlechterdings lässt sich das durch den Gesetzentwurf Erstrebte unseres Erachtens durch die vorgeschlagenen Regelungen in keiner Weise erreichen, da die vorgeschlagenen Regelungen konzeptionell ungenügend durchdacht sind. Insbesondere wird die sich aufdrängende Frage nach standardisierten Übersetzungsvorgaben nicht thematisiert. Die angestrebte Verbreitung des deutschen Rechts ist zudem nicht lediglich in der Verfahrenssprache begründet. Am Ende steht der Gefährdung von Rechtsprechung und Rechtssprache kein adäquater Nutzen gegenüber. Neben der thematisierten problematischen Aufgabe der Einheit von Rechts- und Verfahrenssprache sind zudem auch verfassungsrechtliche Bedenken ins Feld zu führen, die durch die Begründung zum Gesetzentwurf verharmlost werden.

Unseres Erachtens ist die rechtliche Sicherung der deutschen Sprache dort notwendig, wo sie praktische sowie rechtliche und demokratische Funktionen erfüllt, die nicht von anderen Sprachen in gleicher Weise geleistet werden können bzw. wo Mehrsprachigkeit keine zielführende Alternative darstellt. Demgemäß ist gegen eine mehrsprachige deutsche Gesellschaft grundsätzlich kaum etwas einzuwenden, gegen eine Gefährdung der hochsensibel mit Sprache gute Arbeit leistenden staatlichen Gewalt schon. 